\title{
Efecto de la velocidad de deformación en la recristalización dinámica de un cobre ETP durante su compresión en caliente con temperatura descendente ${ }^{(\cdot)}$
}

\author{
G. Torrente*, M. Torres* y L. Sanoja**
}

\begin{abstract}
Resumen
En el presente trabajo se estudia el efecto de la velocidad de deformación en la recristalización dinámica de un cobre electrolítico puro durante su deformación en caliente con temperatura descendente. Para ello, se realizaron ensayos de compresión en caliente hasta deformaciones verdaderas cercanas a uno, con cuatro velocidades de deformación, mientras descendía la temperatura. Los ensayos realizados a las dos velocidades de deformación más bajas mostraron recristalización dinámica de picos múltiples con un ascenso de la tensión en lugar del estado de saturación, debido probablemente al continuo descenso de la temperatura. Con el aumento de la velocidad, los ensayos restantes mostraron recristalización de pico simple y solo restauración, respectivamente. Los resultados experimentales se compararon con los de una simulación basada en el modelo cosenoidal amortiguado de Avrami. La simulación arrojó resultados próximos a los medidos durante la recristalización dinámica de picos múltiples, sugiriendo la aplicación del modelo a procesos de recristalización dinámica de picos múltiples con temperatura variable.
\end{abstract}

Palabras Claves Cobre electrolítico puro; Recristalización de picos múltiples; Modelo cosenoidal amortiguado de Avrami; KJAM; Zener-Hollomon.

\section{Effect of the strain rate in the dynamic recrystallization of ETP copper during its hot compression with descending temperatures}

\begin{abstract}
The main purpose of this project is to establish the effect of strain rate in the dynamic recrystallization of an ETP copper during its hot deformation with descending temperature. For this, there were made some tests of hot compression until true deformations close to one, with four strain rates while the temperature was descending. The tests that were made to the two lowest strain rates, showed a multiple peaks dynamic recrystallization with a rise of the tension instead it reaches the steady state, maybe due a continuous decline of the temperature. With the increase of rate the rest of the tests showed simple peak recrystallization and recovering respectively. The experimental results were compared with the results of a simulation based on the Damped Cosine Avrami Model. The simulation produced results closed to those measured during the multiple peaks dynamic recrystallization. These suggest that the application of this Model may be extended to multiple peaks dynamic recrystallization processes with changeable temperature.
\end{abstract}

Keywords

Electrolytic pure copper; Multiple peaks dynamic recrystallization; Damped cosine Avrami model; KJAM; ZenerHollomon.

\section{INTRODUCCIÓN}

La mayoría de los procesos industriales para la obtención de productos por conformado en caliente transcurren durante el enfriamiento del material que se está trabajando. Esto ocurre debido a que no es posible aislar térmicamente por completo el material de trabajo del equipo, imposibilitando realizar el conformado en condiciones adiabáticas, ya sea en un pro- ceso de colada continua, en trefilado de conductores eléctricos de cobre o en cualquier otro proceso industrial de conformado en caliente.

El conformado en caliente es uno de los métodos más empleados para obtener productos metálicos semielaborados o finales. La recristalización durante el conformado en caliente es un proceso dinámico durante el cual las velocidades relativas de nucleación y crecimiento de granos nuevos determinan las propiedades mecánicas

\footnotetext{
(•) Trabajo recibido el 25 de agosto de 2011 y aceptado en su forma final el día 11 de noviembre de 2011.

* Departamento de Mecánica, Universidad Simón Bolívar, Valle de Sartenejas, 89000 Caracas, Venezuela.

** Coordinación de Ingeniería de Materiales, Universidad Simón Bolívar, Valle de Sartenejas, 89000 Caracas, Venezuela.
} 
del material, definiendo su futura conformabilidad a temperatura ambiente. En el conformado de metales, como por ejemplo en el conformado de conductores de cobre, es importante conocer la relación entre la tensión y la deformación a medida que se procesa en caliente, así como también predecir el tamaño de grano final y las condiciones del material luego de ser deformado.

En los procesos de conformado a elevadas temperaturas el material experimenta tres etapas que definen las propiedades finales del material. La primera es el endurecimiento por deformación el cual depende de los defectos presentes en la estructura, como por ejemplo los bordes de grano, que restringen el movimiento de las dislocaciones y contribuyen al endurecimiento durante la deformación ${ }^{[14]}$. La segunda es la restauración, en donde hay una compensación simultánea entre los mecanismos de endurecimiento y los mecanismos de ablandamiento, como los procesos dinámicos de aniquilación de dislocaciones y formación de sub-granos. La tercera etapa es la recristalización, en donde nuclean y crecen granos nuevos. Actualmente, son aceptados diferentes modelos para predecir el comportamiento de metales durante los procesos dinámicos de recristalización; entre los más difundidos están el modelo de KJMA y las ecuaciones de Lutton y Sellar ${ }^{[1-5]}$.

En 1937, Andrei Kolmogorov ${ }^{[6]}$ desarrolló una teoría para explicar la cinética de la recristalización estática de metales a temperatura constante. En 1939, William Johnson y Robert Meh[ ${ }^{[7]}$ publicaron un estudio sobre los procesos de solidificación, basados en nucleación y crecimiento, el cual coincidía con el presentado por Kolmogorov ${ }^{[6]}$; en este mismo año, Avrami ${ }^{[4]}$ presentó su estudio general sobre la cinética de los cambios de fase. A partir de la unificación de estos trabajos, se conformó la teoría KJAM la cual se ha convertido en el modelo estándar para analizar los procesos de transformación basados en la nucleación y crecimiento.
En 1959, Rossard ${ }^{[24]}$ propuso que uno de los tipos de recristalización dinámica, conocida como recristalización cíclica o de picos múltiples, muestra una relación de la tensión con la deformación de tipo oscilatorio atenuado. En el año 2000, Prado et al. ${ }^{[2,3 \text { y } 8]}$ proponen el Modelo Cosenoidal Amortiguado de Avrami, basado en el modelo de KJAM, como una herramienta para predecir este tipo de comportamiento a temperatura constante.

El objetivo de este trabajo es presentar un estudio preliminar del efecto de la velocidad de deformación sobre la relación entre la tensión y la deformación de un cobre electrolítico puro (ETP) durante un proceso de compresión en caliente con temperatura descendente, a partir de resultados experimentales. Estos resultados se comparan con valores aproximados obtenidos a partir del modelo cosenoidal amortiguado de Avrami, para evaluar el empleo de este modelo como una herramienta para predecir el comportamiento mecánico del cobre en este tipo de proceso. De esta comparación se determina que el modelo cosenoidal amortiguado de Avrami arroja resultados próximos a los resultados experimentales cuando el cobre ETP experimenta un proceso de recristalización dinámica de picos múltiples durante un proceso de compresión en caliente con temperatura descendente.

\section{METODOLOGÍA}

El cobre ETP empleado fue adquirido en forma de barras de 12,6 mm de diámetro, las cuales fueron tratadas térmicamente calentándolas a $850^{\circ} \mathrm{C}$ por $15 \mathrm{~min}$ con un enfriamiento posterior en aire. El material fue caracterizado mediante análisis químico, ensayos mecánicos y observación en microscopía óptica. Para el análisis químico se empleó un espectrofotómetro de emisión atómica, Spectro Lab Junior; los resultados se presentan en la tabla I. El contenido

Tabla I. Composición química del cobre

Table I. Chemical composition of copper

\begin{tabular}{cccccccc}
\hline \multicolumn{7}{c}{$\begin{array}{c}\text { Elementos químicos } \\
\left(\% \text { en peso } \times \mathbf{1 0}^{3}\right)\end{array}$} \\
\hline $\mathrm{Zn}$ & $\mathrm{Pb}$ & $\mathrm{Sn}$ & $\mathbf{P}$ & $\mathbf{M n}$ & $\mathrm{Fe}$ & $\mathbf{N i}$ & $\mathrm{Si}$ \\
3,4 & 14,4 & 4,9 & $<0,4$ & 2,7 & 25,6 & $<0,7$ & 1,2 \\
$\mathrm{Co}$ & $\mathrm{Al}$ & $\mathbf{S}$ & $\mathrm{Be}$ & $\mathbf{Z r}$ & $\mathrm{Au}$ & $\mathrm{Sb}$ & $\mathbf{M g}$ \\
$<1,6$ & 19,9 & $<0,3$ & 0,1 & $<0,3$ & 1,6 & $<3$ & 5,5 \\
& $\mathrm{Cr}$ & $\mathrm{Te}$ & $\mathrm{As}$ & $\mathbf{C d}$ & $\mathrm{Bi}$ & $\mathrm{Ag}$ & \\
& 1,6 & $<5$ & $<0,6$ & $<0,7$ & $<2,5$ & 6,1 & \\
\hline
\end{tabular}

NOTA: Balance al $100 \%$ de Cu. 
de oxígeno presente en el cobre estudiado, no fue determinado.

Los resultados de los ensayos mecánicos a temperatura ambiente y diferentes velocidades de deformación $(\dot{\varepsilon})$ se reportan en la tabla II, donde $\sigma_{y}$ y $\sigma_{u}$ son los esfuerzos verdaderos de fluencia y carga máxima, respectivamente. Estos resultados se obtuvieron a partir de ensayos de compresión realizados en una máquina universal de ensayos, MTS 312.31/227, de 25 toneladas de capacidad. Los ensayos se realizaron según las especificaciones de la norma ASTM E 9-98 ${ }^{[28]}$.

Para estudiar el efecto de la velocidad de deformación sobre el comportamiento mecánico en caliente de un cobre ETP, se ensayaron probetas a compresión empleando velocidades de deformación constantes e iguales a 0,$001 ; 0,01 ; 0,1$ y $1 \mathrm{~s}^{-1}$.

Los ensayos, a las diferentes velocidades, se realizaron por triplicado a probetas cilíndricas de cobre ETP de diámetro $\phi=12,6 \mathrm{~mm}$ y longitud $\mathrm{L}=18,9 \mathrm{~mm}$, con una relación de esbeltez $\mathrm{L} / \phi=1,5$ (de acuerdo a la norma ASTM E209)[26].

Para la realización de los ensayos de compresión en caliente, se diseñaron y construyeron dos mordazas cilíndricas, de $30 \mathrm{~mm}$ de diámetro y $250 \mathrm{~mm}$ de longitud, encamisadas para su refrigeración con circulación de etilenglicol. Las mordazas se fabricaron por mecanizado en acero AISI H-13 y posteriormente fueron templadas, en sales fundidas, y revenidas. Las mordazas fueron instaladas en la máquina universal de ensayos mecánicos MTS $312.31 / 227$, de 25 toneladas de capacidad. La adquisición de datos de los ensayos se llevó a cabo a razón de 500 puntos por milímetro de desplazamiento, empleando el programa DASYLab. Para el calentamiento de las muestras, durante los ensayos de compresión, se empleó un horno portátil de resistencia eléctrica ATS® 2961 acoplado a la máquina universal de ensayos (Fig. 1).

En todos los ensayos se empleó polvo de grafito como lubricante entre las muestras de cobre y las mordazas.

Tabla II. Valores reales de los esfuerzos de fluencia y máximo del cobre estudiado, a temperatura ambiente

Table II. Yield stress and ultimate stress of studied copper at room temperature

\begin{tabular}{ccc}
\hline$\dot{\varepsilon}\left(\mathbf{s}^{-1}\right)$ & $\sigma_{\mathbf{y}}(\mathrm{MPa})$ & $\sigma_{\mathrm{u}}(\mathrm{MPa})$ \\
\hline 0,001 & 117 & 277 \\
0,01 & 103 & 308 \\
0,1 & 135 & 304 \\
1 & 127 & 305 \\
\hline
\end{tabular}

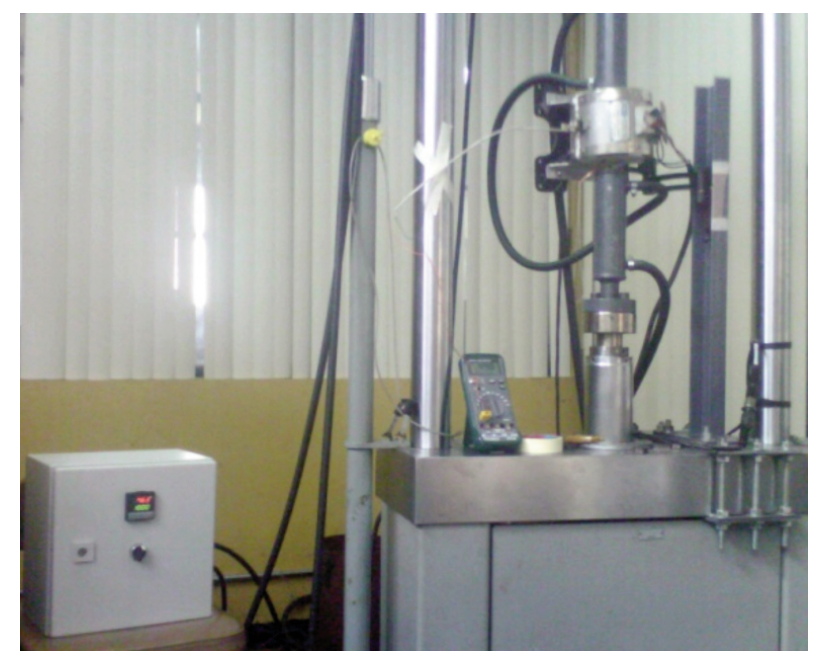

Figura 1. Montaje experimental: horno ATS $®$ 2961, mordazas, sistema de medición y control de temperaturas, para el horno y la probeta, y máquina universal de ensayos mecánicos MTS $®$ $312.31 / 227$.

Figure 1. Experimental set: furnace ATS $₫ 2961$, jaws, temperature monitoring system of the furnace and the specimens and universal machine for mechanical testing MTS ${ }^{\circledR} 312.31 / 227$.

El procedimiento seguido para la realización de cada uno de los ensayos fue el siguiente: 1) El horno se calentó hasta alcanzar una temperatura de $540+/-8^{\circ} \mathrm{C}$; 2) se introdujo la probeta de cobre en el interior del horno y se esperó un tiempo de aproximadamente $20 \mathrm{~min}$ para estabilizar la temperatura, obteniéndose un tamaño promedio de grano de $29 \mu \mathrm{m}$; y 3 ) las mordazas se aproximaron a la probeta dirigiendo manualmente el equipo hasta hacer un ligero contacto con ellas e inmediatamente se inició el ensayo. Durante todo el ensayo se midió la temperatura de la muestra, registrando su descenso para cada velocidad de deformación estudiada. Una vez alcanzada una deformación verdadera cercana a 1 , se finalizó el ensayo y la probeta de cobre se templó en agua para preservar su estructura cristalina. Los diámetros de todas las probetas ensayadas se midieron longitudinalmente (en dirección paralela a la carga aplicada) registrándose una variación promedio máxima igual a $13 \%$, debido al abarrilamiento.

Para la determinación de las curvas verdaderas tensión-deformación, se realizaron dos correcciones pertinentes. La primera de ellas relacionada con el desplazamiento real $\left(\Delta l_{\text {real }}\right)$ para obtener la deformación real a compresión (e) empleando ecuaciones estándares. Este desplazamiento se obtuvo restándole al desplazamiento experimental $\left(\Delta l_{\text {exp }}\right)$, el desplazamiento al inicio del ensayo para corregir la curvatura 
inicial de la zona elástica por el ajuste de contacto entre elementos $\left(\Delta l_{\text {inicial }}\right)$ y el debido a la deflexión elástica del equipo $\left(\Delta l_{\text {equipo }}\right)$. La deflexión elástica del equipo se obtuvo a partir de la recta ajustada en la gráfica de la figura 2. Esta gráfica muestra la relación carga-desplazamiento obtenida a partir del ensayo de compresión realizado a las mordazas a $500^{\circ} \mathrm{C}$.

La segunda corrección considera la fricción entre las mordazas y la probeta para obtener las tensiones verdaderas $(\sigma)$, a partir de las tensiones experimentales $\left(\sigma_{E}\right)$ de los ensayos de compresión, empleando el principio de conservación de volumen. Los valores de las tensiones verdaderas se obtuvieron tomando en cuenta el efecto de la fricción, a partir de la ecuación (1) ${ }^{[21]}$.

$$
\sigma_{E}=\sigma \frac{\exp \left(2 \mu \frac{l}{h}\right)-2 \mu \frac{l}{h}-1}{2\left(\mu \frac{l}{h}\right)^{2}}
$$

En esta ecuación, $\mu$ es el coeficiente de fricción, $d$ y $l$ son el diámetro y la longitud instantánea de la probeta, respectivamente. En este trabajo se empleó un valor para el coeficiente de fricción entre el grafito y el cobre igual a $0,05^{[29}$ y 30$]$.

Una vez realizadas las correcciones señaladas, con los valores verdaderos se graficaron las curvas de tensión en función de la deformación para el cobre estudiado.

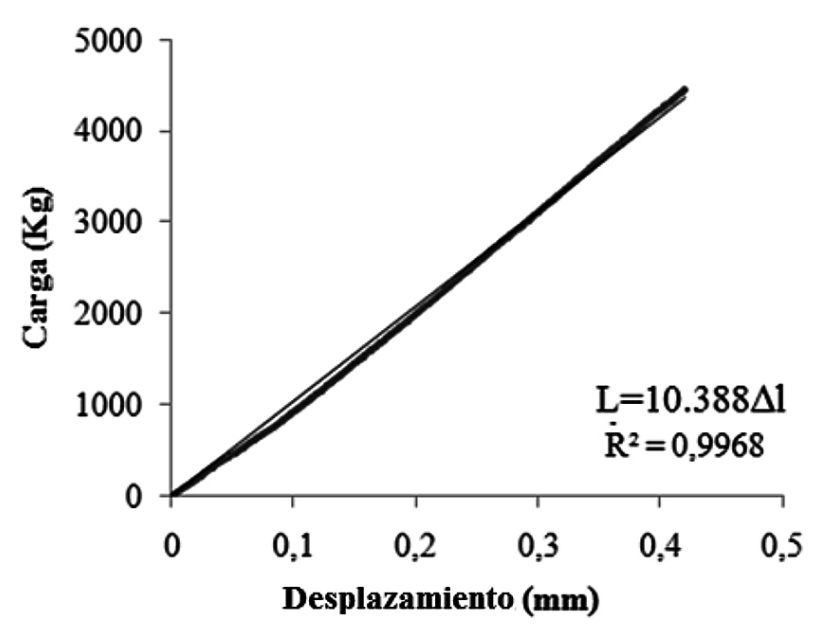

Figura 2. Comportamiento elástico del equipo a $500{ }^{\circ} \mathrm{C}$.

Figure 2. Elastic behavior of the equipment at $500^{\circ} \mathrm{C}$.

Para determinar el descenso de la temperatura en el cobre durante los ensayos, se graficaron los valores de temperatura registrados junto a las curvas de tensión en función de la deformación, como se observa en la figura 3, para la velocidad de deformación igual a 0,001 s $\mathrm{s}^{-1}$. El comportamiento de las curvas de temperatura se ajustó para obtener la mejor tendencia y las ecuaciones resultantes, para todas las velocidades de deformación empleadas, se muestran en la tabla III.

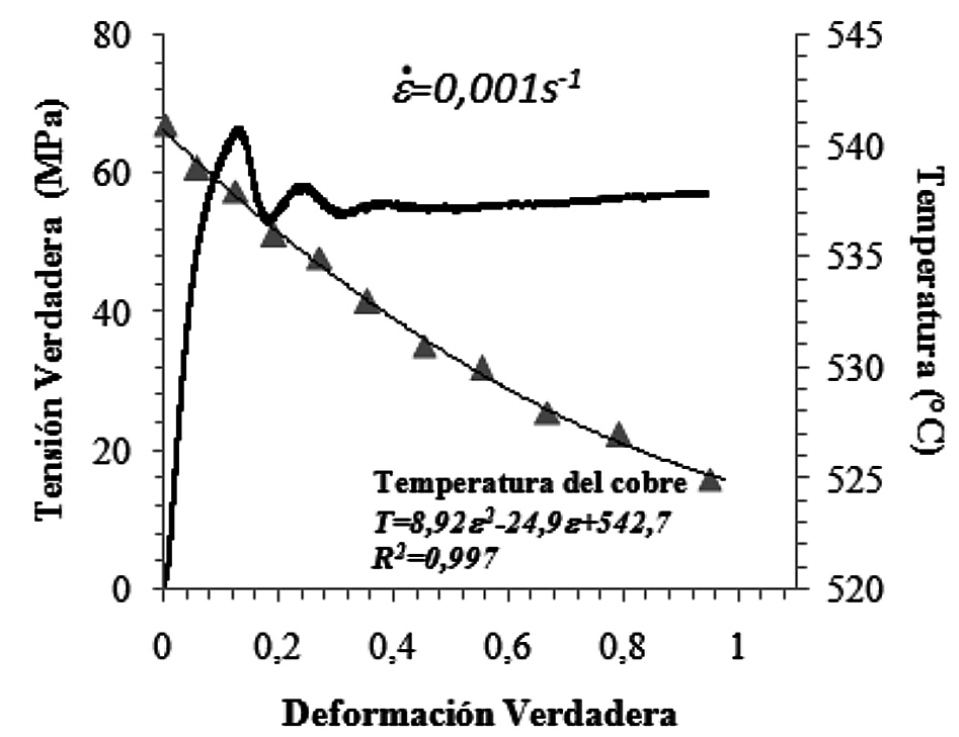

Figura 3. Comportamiento del cobre a compresión, con una velocidad de deformación de $=0,001 \mathrm{~s}^{-1}$, y del descenso de la temperatura durante el ensayo.

Figure 3. Behavior of copper being compressed with strain rate of $=0.001 \mathrm{~s}^{-1}$ while cooling. 
EFECTO DE LA VELOCIDAD DE DEFORMACIÓN EN LA RECRISTALIZACIÓN DINÁMICA DE UN COBRE ETP DURANTE SU COMPRESIÓN EN CALIENTE CON TEMPERATURA DESCENDENTE EFFECT OF THE STRAIN RATE IN THE DYNAMIC RECRYSTALLIZATION OF ETP COPPER DURING ITS HOT COMPRESSION WITH DESCENDING TEMPERATURES

Tabla III. Temperatura experimental de las probetas en función de la deformación verdadera

Table III. Experimental measure of copper specimens' temperatures like a function of real strain

\begin{tabular}{ccc}
\hline$\dot{\varepsilon}\left(\mathbf{s}^{-1}\right)$ & $\begin{array}{c}\text { Temperatura }\left({ }^{\circ} \mathrm{C}\right) \text { de las probetas } \\
\text { en función de la deformación }\end{array}$ & $\mathbf{R}^{2}$ \\
\hline 0,001 & $\mathrm{~T}=8,92 \varepsilon^{2}-24,9 \varepsilon+542,77$ & 0,997 \\
0,01 & $\mathrm{~T}=8,920 \varepsilon^{2}-24,9 \varepsilon+540,77$ & 0,997 \\
0,1 & $\mathrm{~T}=-20,707 \varepsilon+548,01$ & 1,000 \\
1 & $\mathrm{~T}=-17,044 \varepsilon+532,03$ & 1,000 \\
\hline
\end{tabular}

Para complementar el estudio, las probetas ensayadas se cortaron en dirección paralela a la carga aplicada y sus superficies se prepararon metalográficamente siguiendo las recomendaciones de las normas ASTM E3-11 [27]. Posteriormente, estas superficies se atacaron químicamente, empleando una solución compuesta por $10 \mathrm{ml}$ de $\mathrm{HNO}_{3}$ y $90 \mathrm{ml}$ de $\mathrm{H}_{2} \mathrm{O}$, para su observación a través de microscopía óptica. Esta observación permitió la toma de fotomicrografías y la determinación del tamaño de grano recristalizado, a partir del método de intercepto de Heyn, según las recomendaciones de la norma ASTM E 112-88 ${ }^{[31]}$.

Una vez obtenidos los resultados experimentales, se procedió a compararlos con los resultados obtenidos del modelo cosenoidal amortiguado de Avrami y de la relación de Derby.

\section{MODELO COSENOIDAL AMORTIGUADO DE AVRAMI}

La aplicación del modelo cosenoidal amortiguado de Avrami para describir la recristalización dinámica del cobre, fue propuesto por García et al. ${ }^{[6,2, \text { y } 3]}$. Este modelo se basa en la teoría de KJMA.

La mayoría de los modelos propuestos para describir el proceso de recristalización dinámica, incluyendo el modelo cosenoidal amortiguado, se basan en el parámetro de Zener-Hollomon $Z$ de acuerdo a la ecuación $(2)^{[3,6 \text { y } 9]}$.

$$
Z=\dot{\varepsilon} \cdot \exp \left(\frac{Q_{s d}}{R T}\right)
$$

En esta ecuación, $R$ es la constante de gases ideales; $T$ es la temperatura que, en este trabajo, corres- ponde a la temperatura instantánea; $y \mathrm{Q}_{s d}$ es la energía de activación aparente del cobre ${ }^{[10]}$.

La energía de activación aparente para el cobre debe ser más baja que su energía de autodifusión $(195 \mathrm{KJ} / \mathrm{mol})^{[10]}$ y más alta que su energía para la difusión del borde de grano $(85 \mathrm{KJ} / \mathrm{mol})^{[10]}$. En este trabajo se empleó un valor de $Q_{s d}=180 \mathrm{KJ} / \mathrm{mol}$ en el modelo cosenoidal amortiguado, dado que con este valor se obtuvieron resultados próximos a los obtenidos experimentalmente. Este valor no se aleja del empleado por Prasad et al..$^{[10]}$ en su investigación, relacionada con los mecanismos de deformación a altas temperaturas en un cobre electrolítico, el cual fue de $Q_{s d}=159+/-10 \mathrm{KJ} / \mathrm{mol}$, para temperaturas entre 400 y $600{ }^{\circ} \mathrm{C}$ y velocidades de deformación entre 0,001 y $1 \mathrm{~s}^{-1}$. Esta diferencia puede deberse a que probablemente el contenido de oxígeno presente en el cobre estudiado por Prasat et al. ${ }^{[10]}$ es diferente al del cobre estudiado en este trabajo. Al respecto, Prasat et al. ${ }^{[10]}$ señalan que el valor de la energía aparente de activación del cobre electrolítico aumenta con el contenido de oxígeno, debido al aumento de las partículas de óxido de cobre en la matriz. Este comportamiento, se observa para temperaturas entre 400 y $950{ }^{\circ} \mathrm{C}$ y velocidades de deformación entre 0,001 y $100 \mathrm{~s}^{-1}$ [10].

Con el valor de Zener-Hollomon es posible determinar diversos valores característicos que describen los procesos de recristalización dinámica, como por ejemplo el valor de la deformación pico, $\varepsilon_{\mathrm{P}}$, y tensión pico, $\sigma_{P}$, que definen el punto máximo de tensión, de acuerdo a las ecuaciones (3) y (4).

$$
\begin{gathered}
\varepsilon_{P}=k_{P} \phi_{0}^{N_{d}} Z^{N_{E}} \\
\sigma_{P}=\sigma_{r}+\sigma_{d}
\end{gathered}
$$

La deformación pico es función de $K_{p}, N_{d}$ y $N_{E}$, las cuales son constantes que dependen del mate- 
rial ${ }^{[19]}$, siendo ésta directamente proporcional al diámetro inicial de grano de la muestra $\phi_{0}$, tal como lo observó Blaz et al. ${ }^{[11]}$.

Para determinar la tensión pico es necesario sumar el máximo valor de la tensión oscilatoria, $\sigma_{d}$, y la contribución de la tensión complementaria, también conocida como amplitud inicial $\sigma_{r}^{[11]}$, la cual es despreciable para recristalizaciones de pico simple. Adicionalmente, la deformación pico está relacionada proporcionalmente a la deformación crítica $\left(\varepsilon_{C}\right)$, la cual es el punto de inflexión que marca la transición entre los procesos dinámicos de restauración y recristalización ${ }^{[8]}$, siendo para el cobre $\varepsilon_{\mathrm{C}} / \varepsilon_{\mathrm{P}} \approx 0,65$, para toda velocidad de deformación a $400^{\circ} \mathrm{C}[12]$. Es importante destacar que el modelo cosenoidal amortiguado de Avrami supone la deformación pico como la transición entre los procesos dinámicos de restauración y recristalización $\left.{ }^{[8 \text { y }} 16\right]$.

Por otro lado, la relación entre la tensión y la deformación en los procesos dinámicos de recuperación se puede representar a partir de relaciones que involucran tanto la deformación pico como la deformación crítica. En particular, esta relación para los procesos dinámicos de endurecimiento y restauración puede ser descrita por la ecuación (5) ${ }^{[1 \text { y } 13]}$.

$$
\sigma=\sigma_{S S}+\left(\sigma_{i}-\sigma_{S S}\right)\left[\exp \left(-\frac{\varepsilon_{P}-\varepsilon_{i}}{\varepsilon_{C}}\right)\right]
$$

En este trabajo se tomó el origen como valor de referencia $\left(\varepsilon_{i}=0 ; \sigma_{i}=0\right)$ para determinar el comportamiento en las etapas de endurecimiento y restauración (Ec. (5)). La tensión de compresión en estado estacionario, $\sigma_{S S}$, se puede representar como una función del máximo valor de la tensión oscilatoria, $\sigma_{d}$, y de la tensión de ablandamiento, $\sigma_{f}$, según la ecuación (6).

$$
\sigma_{s s}=\sigma_{d}-\sigma_{f}
$$

El proceso dinámico de recristalización se inicia cuando la deformación supera la deformación crítica y puede ser de pico simple o de picos múltiples. Con el valor de $Z$ es posible predecir si el cobre presentará una recristalización dinámica de pico simple (cuando $Z>Z_{C}$ ) o picos múltiples (cuando $Z<Z_{C}$ ). El valor de referencia $Z_{C}$, conocido como Zener-Hollomon crítico, es una función del diámetro inicial de grano del material en estudio. Esta relación entre el diámetro inicial de grano y el valor de $Z_{C}$ fue observada experimentalmente por Sakui et al. ${ }^{[32]}$, en el año 1977, para un acero con 0,16 \% C. Posteriormente, en el año 1996, Montheillet et al. ${ }^{[33]}$ observaron que el valor de $Z_{C}$ tiende a un comportamiento potencial negativo con el tamaño de grano, aumentando el valor de $Z_{C}$ en la medida que el tamaño inicial de grano es menor, favoreciéndose la recristalización dinámica de picos múltiples para granos pequeños en comparación con granos de mayor diámetro, para unas mismas condiciones de velocidad de deformación y temperatura.

En el caso de recristalización dinámica de pico simple, el comportamiento mecánico considerando la tensión se describe con la ecuación (7), de tipo Avrami ${ }^{[2}$ y 3$]$ :

$$
\sigma=\sigma_{P}-\left(\sigma_{P}-\sigma_{S S}\right) \cdot\left\{1-\exp \left[-0,693\left(\frac{\varepsilon-\varepsilon_{P}}{\dot{\varepsilon} \cdot t_{50 \%}}\right)^{n}\right]\right\}
$$

En la ecuación (7), $n$ es una función de $Z$ y $t_{50 \%}$ es el tiempo necesario para que recristalice el $50 \%$ de volumen del material; la expresión entre llaves corresponde a la fracción volumétrica recristalizada ${ }^{[23]}$, la cual es el pilar de la teoría KJMA ${ }^{[6 \text { y } 7]}$. Esta ecuación se fundamenta en las siguientes hipótesis ${ }^{[15]}$ :

- La nucleación debe ocurrir de forma aleatoria y homogénea sobre toda la fase no transformada.

- La velocidad de crecimiento no se debe afectar por la extensión de la transformación.

- La velocidad de crecimiento debe ser la misma en todas direcciones.

Para el valor de n, en la ecuación (7), García et al. ${ }^{[2,3,16 \text { y } 17]}$ propusieron una función de $Z$, donde $n=3,69-0,221 \operatorname{Ln}(Z)$ y el tiempo necesario para que recristalice el $50 \%$ de volumen del material es:

$$
t_{50 \%}=0,001 \cdot \phi_{0}^{0,4} \cdot \dot{\varepsilon} \cdot \exp \left(\frac{Q}{R T}\right)
$$

En la ecuación (8), Q es la energía de activación para el control de la recristalización del cobre con

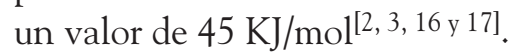

Para el caso de una recristalización dinámica de picos múltiples, la tensión en función de la deformación se comportaría de la siguiente forma:

$$
\begin{aligned}
& \sigma=\sigma_{r} \cdot \exp \left[-S\left(\varepsilon-\varepsilon_{P}\right)\right] \cdot \cos \left[\gamma\left(\varepsilon-\varepsilon_{P}\right)\right] \\
& +\sigma_{d}-\sigma_{f}\left\{1-\exp \left[-D_{g}\left(\varepsilon-\varepsilon_{P}\right)\right]\right\}
\end{aligned}
$$

Los valores de amplitud inicial $\sigma_{r}$, atenuación $S$, el coeficiente de longitud de onda $\gamma$, el coefi- 
EFECTO DE LA VELOCIDAD DE DEFORMACIÓN EN LA RECRISTALIZACIÓN DINÁMICA DE UN COBRE ETP DURANTE SU COMPRESIÓN EN CALIENTE CON TEMPERATURA DESCENDENTE EFFECT OF THE STRAIN RATE IN THE DYNAMIC RECRYSTALLIZATION OF ETP COPPER DURING ITS HOT COMPRESSION WITH DESCENDING TEMPERATURES

Tabla IV. Relaciones Empíricas

Table IV. Empirical Relations

$\sigma_{r}[M P a]=-1,68 \times 10^{-2} \cdot \operatorname{Ln}(Z)+4,74$
Para $Z<Z_{C}:$
$\sigma_{d}[M P a]=0,815 \cdot Z^{0,22}$
Para $Z>Z_{C}:$
$\sigma_{d}[M P a]=2,3\left(\frac{E(T)}{1204,2}\right) \cdot \sinh ^{-1}\left(328\left[\left(\frac{\dot{\varepsilon}}{D(T)}\right)^{1 / 5}\right]\right)$
$E(T)=111986\left[1-0,54\left(\frac{T}{1356}\right)\right]$
$D(T)=2 \times 10^{-5} \cdot \exp \left(\frac{Q_{s d}}{R T}\right)$
$\sigma_{f}[M P a]=0,143 \cdot Z^{0,21}$
$\gamma=7252,69 \cdot Z^{-0,24}$
$D_{g}=0,89 \cdot \log (Z)+6,71$
$S=-4,36 \cdot \operatorname{Ln}(Z)+37,65$
$\varepsilon_{P}=3,3 \times 10^{-4} \cdot Z^{0,29}$
$\varepsilon_{C}=1,128 \cdot 10^{-2} \cdot Z^{0,1113}$
$*(16)$

ciente de descenso $D_{g}$, la constante de Young $E(T)$ y el coeficiente de autodifusión $D(T)$ son valores empíricos que, en su mayoría, son funciones dependientes de $Z$ o T. Estas funciones se muestran en la tabla IV.

Las ecuaciones empíricas mostradas en la tabla IV fueron tomadas del trabajo de García et al.[2, 3, 16 y 17], excepto los parámetros de las ecuaciones identificadas con $(*)$, los cuales fueron ajustados con los resultados experimentales de este trabajo siguiendo el procedimiento recomendado por Cabrera ${ }^{[18]}$.

Para complementar los resultados obtenidos de la simulación, se empleó la ecuación de Derby ${ }^{[19}$ y 20] para estimar el diámetro de grano recristalizado (Ec. (21)). Esta ecuación relaciona el esfuerzo en estado estacionario $\sigma_{s s}$, el vector de burguer $b$, el diámetro de grano recristalizado $D_{\text {rex }}$, el coeficiente de cizalladura $\mathrm{m}(\mathrm{T})$ y el exponente de Derby " $n_{\text {Derby }}$ " con la constante de Derby
"K $K_{\text {Derby }}$ ". En este trabajo se tomaron los valores propuestos por Derby ${ }^{[20]}$ en su trabajo, de $2 / 3$ y 3,7 para el exponente y la constante de Derby, respectivamente $[8,16$ y 20].

Los valores de diámetros de granos recristalizados estimados con la ecuación de Derby se compararon con los valores experimentales, obtenidos del estudio metalográfico del cobre comprimido.

$$
\frac{\sigma_{S S}}{\mu(T)}\left(\frac{D_{\text {rex }}}{b}\right)^{n_{\text {Derby }}}=K_{\text {Derby }}
$$

\section{RESULTADOS}

Para establecer el efecto de la velocidad de deformación sobre el comportamiento mecánico a com- 


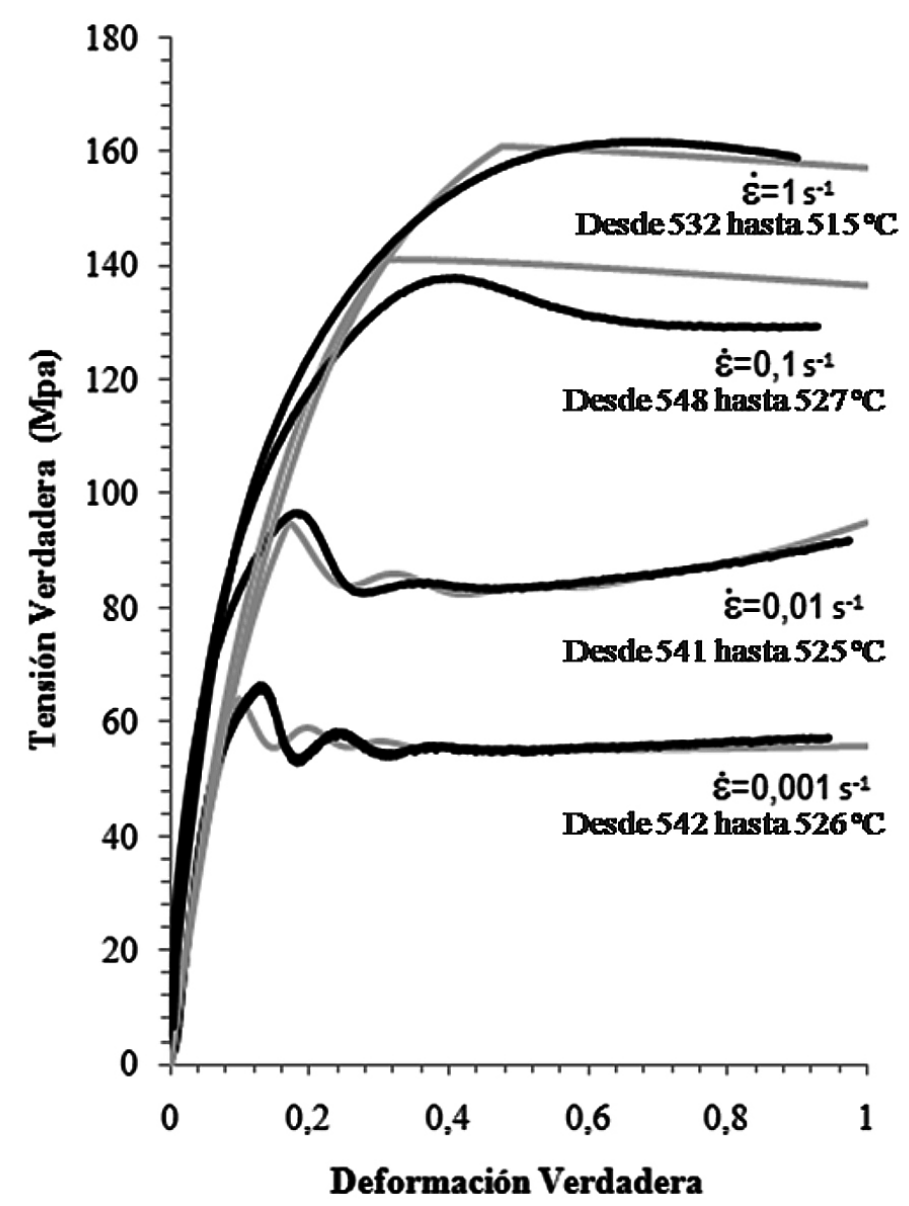

Figura 4. Curvas verdaderas de tensión en función de la deformación en caliente, con descenso de temperatura en los intervalos indicados. Las líneas de color negro representan los valores experimentales y las líneas en gris los valores simulados.

Figure 4. Real stress vs. real strain curves of hot compression tests with decreasing temperature like show table I (black line experimental results, gray line simulation results).

presión en caliente del cobre, con temperatura descendente, en la figura 4 se muestran las curvas verdaderas de tensión en función de la deformación experimentales (con líneas negras) y simuladas (con líneas grises), para las 4 velocidades de deformación. También se indican, para cada velocidad de deformación, las temperaturas inicial y final, que muestran su descenso.

En la figura 4, las curvas simuladas se obtuvieron empleando las ecuaciones de la (1) a la (20) que conforman el modelo cosenoidal amortiguado de Avrami, conjuntamente con las ecuaciones que describen el descenso de temperatura para cada velocidad.

Al observar las curvas mostradas en la figura 4 se aprecia un aumento del punto pico con la velocidad de deformación. Este comportamiento ya ha sido observado en anteriores investigaciones ${ }^{[2,3,10}$ y 18] $y$ se describe en las ecuaciones (3) y (4), donde la tensión pico y la deformación pico son proporcionales a $Z$ y por ende a la velocidad de deformación (Ec. (1)).

Por otro lado, en la figura 4, todas las curvas evidencian características propias de un proceso dinámico de recristalización. En ellas se observa también un cambio de comportamiento, de picos múltiples para velocidades de $0,001 \mathrm{~s}^{-1}$ y $0,01 \mathrm{~s}^{-1}$, a pico simple para una velocidad de deformación de $0,1 \mathrm{~s}^{-1}$. Si se toma que la energía aparente de activación $Q_{s d}$ (Ec. (1)) tiene un valor de $180 \mathrm{KJ} / \mathrm{mol}$ para el cobre, el valor de $Z_{C}$ para este cambio de comportamiento, debe estar en un rango entre 7,3594*109 $\mathrm{s}^{-1}<Z_{C}<23,9251 * 10^{9} \mathrm{~s}^{-1}$, que son los valores de Zener Hollomon para 0,01 y 0,1 s-1 respec-

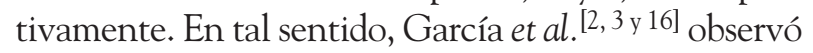
en su trabajo un comportamiento de recristalización dinámica de pico simple para un cobre de 99,95\% de pureza, con un diámetro inicial de grano de $530 \mu \mathrm{m}$, 
deformado a una velocidad de $0,001 \mathrm{~s}^{-1}$ y a $600{ }^{\circ} \mathrm{C}$. Esto indica que se puede conseguir una recristalización con características de pico múltiple en granos más pequeños a la misma velocidad de deformación y temperatura menor que en granos de mayor diámetro, tal como lo observó Sakui et al..$^{[16,28 \text { y } 30]}$ para el acero en el año 1977; por lo que posiblemente también se pueda establecer para el cobre una relación inversamente proporcional entre $Z_{C}$ y el tamaño inicial de grano.

Prasad et al. ${ }^{[10]}$ en su trabajo menciona que a $500^{\circ} \mathrm{C}$ y a velocidades de deformación entre 1 y $10 \mathrm{~s}^{-1}$ el comportamiento del cobre ETP es típico de una recristalización de pico simple; mientras que a velocidades de deformación de $0,1 \mathrm{~s}^{-1}$ o menores la recristalización es de pico múltiple. Estos resultados experimentales de Prasad ${ }^{[10]}$ son comparables a los obtenidos en este trabajo, observándose una diferencia de apenas $2 \mathrm{MPa}$ en los valores del esfuerzo pico para los ensayos realizados a $0,001 \mathrm{~s}^{-1}$.

\subsection{Recristalización dinámica de picos múltiples}

Para las velocidades de deformación de 0,001 y $0,01 s^{-1}$, las curvas experimentales de tensión en función de la deformación presentaron un comportamiento característico de recristalización de pico múltiple. En ellas se observa un endurecimiento del cobre a pequeñas deformaciones, seguido de una zona de transición evidenciado por un cambio de pendiente con una disminución de la velocidad de endurecimiento hasta alcanzar la deformación pico, a partir de la cual se aprecia un comportamiento oscilatorio de la tensión en función de la deformación.

Este comportamiento oscilatorio generalmente es producto de ciclos de recristalización en sincronía, donde cada valle es el inicio de un nuevo ciclo de recristalización. Posteriormente, esta sincronía se va perdiendo, reflejándose en la paulatina atenuación de las oscilaciones, como se aprecia en la figura 4.

La atenuación de las oscilaciones en las curvas con comportamiento de pico múltiple también se observa en las curvas obtenidas del modelo cosenoidal amortiguado de Avrami, lo cual muestra un avance de este modelo en comparación con los anteriores no amortiguados, como los de KJAM, Rossard ${ }^{[24]}$, y Estrin, Mecking y Bergström ${ }^{[25]}$, al tomar en cuenta la atenuación S (Ec. (18)) en el cálculo de la tensión (Ec. (9)).

El leve desplazamiento en la oscilación que se aprecia en los resultados simulados, en comparación con los experimentales (Fig. 4), pudiera deberse a leves diferencias en la precarga aplicada a las probetas debido a la precisión del equipo, lo cual afecta levemente la aproximación empírica de los valores en la ecuación para determinar la deformación pico (Ec. (19)).

A medida que aumenta la deformación $(\varepsilon)$, la diferencia entre ésta y la deformación pico $\left(\varepsilon-\varepsilon_{\mathrm{P}}\right)$ es mayor (Ec. (7)), haciendo que la tensión $(\sigma)$ tienda hacia su valor en estado estacionario, el cual no es un valor constante para las condiciones estudiadas. Esto se observa en los resultados obtenidos del modelo y también en las curvas experimentales.

Un resultado interesante de los ensayos donde ocurrió recristalización a temperatura descendente, es que, después de la desincronización de los ciclos de recristalización, hubo un aumento de la tensión probablemente debido al descenso de la temperatura. Este incremento en la tensión luego de alcanzado el estado estacionario no se observa en ensayos de recristalización dinámica con temperatura constante. Este comportamiento observado en ensayos con temperatura descendente también lo refleja el modelo cosenoidal amortiguado de Avrami, lo cual indica que un ajuste de este modelo permitiría su aplicación a procesos de recristalización dinámica que transcurren con temperatura variable. Aunque no se mantenga un valor constante de la tensión para el estado estacionario, se seguirá empleando el término tensión en estado estacionario para referirse a $\sigma_{s s}$, ya que se alcanza la desincronización en los ciclos de recristalización, característica de este estado.

Por otro lado, los resultados experimentales muestran que la longitud de onda disminuye con la velocidad de deformación, siendo estos dos valores directamente proporcionales, lo cual también se refleja en los resultados del modelo.

\subsection{Recristalización dinámica de pico simple}

En la figura 4 se observa que, para la velocidad de deformación de $0,1 \mathrm{~s}^{-1}$, la curva de fluencia experimental presenta un comportamiento característico de recristalización de pico simple. En ella se aprecia un endurecimiento del cobre a pequeñas deformaciones hasta alcanzar la deformación pico, luego de lo cual se inicia el descenso de la tensión hasta alcanzar la tensión en estado estacionario en donde, al igual que para ensayos a temperatura constante, la tensión se mantiene invariable, por lo menos hasta una deformación verdadera igual a 1 .

Los resultados de tensión en función de la deformación obtenidos del modelo cosenoidal amorti- 
guado de Avrami para el caso de recristalización de pico simple, se desvían levemente de los resultados experimentales. Una de las posibles razones es suponer el punto pico como punto de transición y no el punto crítico, ya que para procesos de recristalización de pico simple la diferencia entre estos dos puntos es mayor que en la recristalización de picos múltiples ${ }^{[8 \text { y } 16]}$, como se observa en la figura 4 . Se han propuesto otras simulaciones para describir la recristalización de pico simple, basadas en el modelo de KJAM, que se ajustan mejor a los resultados experimentales de metales no ferrosos, como la empleada por Zeng et al. ${ }^{[22]}$ para describir este tipo de recristalización en aleaciones de magnesio.

Para una velocidad de deformación igual a $1 \mathrm{~s}^{-1} \mathrm{y}$ hasta una deformación verdadera igual a 1, la curva mostrada en la figura 4 presenta evidencias de una incipiente recristalización. En ella se observa un endurecimiento del cobre a bajas deformaciones, seguido de una zona de transición y una leve disminución de la tensión debida probablemente a una incipiente recristalización.

Al comparar los resultados experimentales obtenidos en el presente trabajo con los presentados por García et al. ${ }^{[2,3,16 y 17]}$ es interesante resaltar ciertas diferencias. Ellos obtuvieron una relación empírica entre la deformación pico y $\mathrm{Z}$ igual a $\boldsymbol{\varepsilon}_{\mathrm{p}}=3,149 \cdot 10^{-3} Z^{0,24}$, para un cobre deformado con velocidades entre 0,001 y $0,3 \mathrm{~s}^{-1}$ a temperaturas entre 600 y $950{ }^{\circ} \mathrm{C}$. En este trabajo se obtuvo la relación dada por $\varepsilon_{P}=3,3 \cdot 10^{-4} Z^{0,29}$, para un cobre ETP de $29 \mu \mathrm{m}$ de diámetro inicial de grano, con 99,94 \% de pureza. La discrepancia entre los valores obtenidos empleando ambas relaciones puede deberse principalmente a la diferencia en el tamaño inicial de grano, ya que a medida que éste es mayor la deformación pico aumenta y se retarda el inicio del proceso de recristalización, tal como lo notó Blaz ${ }^{[11]}$ y Omar ${ }^{[19]}$.

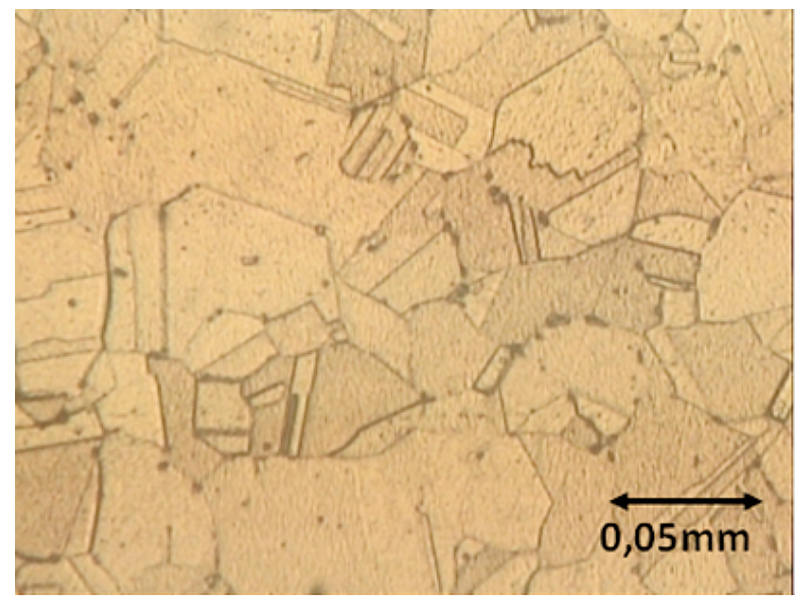

Figura 5. Fotomicrografía del cobre antes de ser comprimido en caliente.

Figure 5. Photomicrograph of copper before hot compression.
Luego de realizados los ensayos de compresión hasta una deformación igual a 1 , las probetas fueron templadas con el propósito de conservar la microestructura de la recristalización dinámica, y comparar sus tamaños de grano con los obtenidos de la ecuación de Derby. Las fotomicrografías de las figuras 5, 6 y 7 muestran las características microestructurales para algunas de las condiciones estudiadas.

Al observar la figura 6 se aprecia un grano no achatado, con geometría poligonal y de mayor

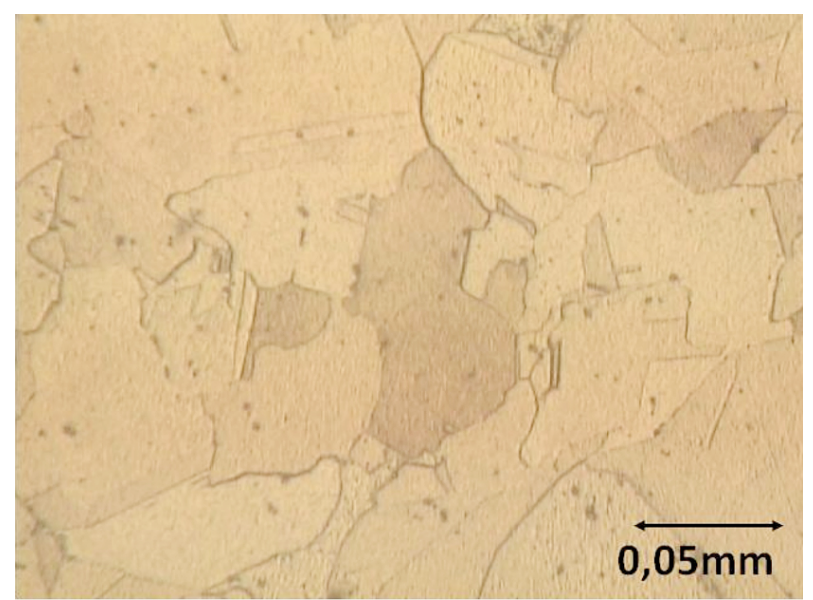

Figura 6. Fotomicrografía del cobre sometido a compresión en caliente a una velocidad de deformación de $0,001 \mathrm{~s}^{-1}$.

Figure 6. Photomicrograph of hot compressed copper with strain rate of $0.001 \mathrm{~s}^{-1}$.

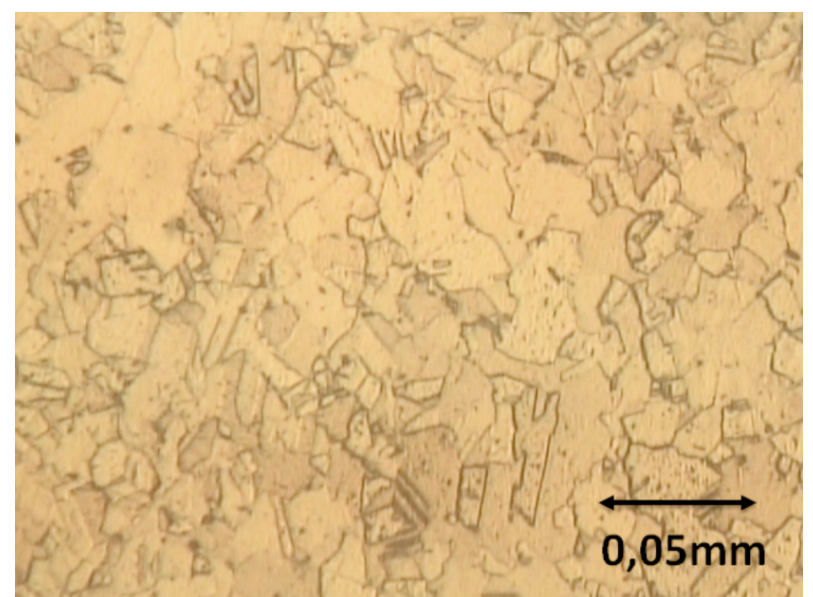

Figura 7. Fotomicrografía del cobre sometido a compresión en caliente a una velocidad de deformación de $0,1 \mathrm{~s}^{-1}$. El tamaño de grano disminuye con el incremento de la velocidad de deformación.

Figure 7. Photomicrograph of hot compressed copper with strain rate of $0.1 \mathrm{~s}^{-1}$. The grain size decreased with rising strain rate. 


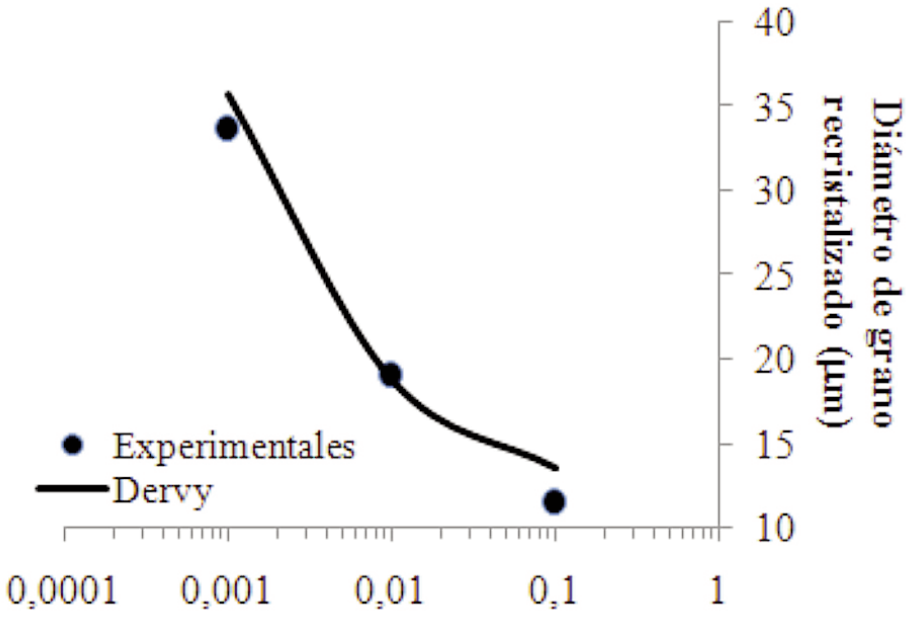

Velocidad de deformación $\left(\mathrm{s}^{-1}\right)$

Figura 8. Comparación entre el tamaño de grano recristalizado medido experimentalmente con el determinado con la ecuación de Derby.

Figure 8. Comparison between the recrystallized grain size measured experimentally and the grain size determined with Derby equation.

tamaño $(33,7 \mu \mathrm{m})$ que su predecesor de la figura 5 $(29 \mu \mathrm{m})$, lo cual muestra que los granos de la figura 6 posiblemente corresponden a nuevos granos que nuclearon y crecieron durante la recristalización. También se observa una disminución de maclas con respecto a la figura 5 , posiblemente como resultado de su aniquilación durante la etapa de restauración.

La figura 7 corresponde a la microestructura de la muestra ensayada a una velocidad de deformación de $0,1 \mathrm{~s}^{-1}$. En ella se aprecia un menor tamaño de grano $(11,56 \mu \mathrm{m})$ en comparación con el de las dos fotomicrografías anteriores. Esto muestra que efectivamente los granos observados en ella corresponden a nuevos granos que nuclearon y crecieron durante el proceso de recristalización, ofreciendo la posibilidad de refinar el grano controlando la velocidad de deformación del proceso de recristalización dinámica.

En la figura 8 se presenta la curva obtenida a partir de la ecuación de Derby y los diámetros de granos medidos experimentalmente, mostrando el comportamiento del diámetro de grano recristalizado en función de la velocidad de deformación. En esta figura se observa una disminución en el tamaño del grano recristalizado a medida que el proceso se realiza a mayores velocidades de deformación.

Para las velocidades de deformación estudiadas, la diferencia máxima registrada entre los valores experimentales y los obtenidos a partir de la relación de Derby, fue de apenas un 5,9\% (Fig. 8). Esta diferencia se observó para una velocidad de deformación igual a $0,001 \mathrm{~s}^{-1}$, donde el valor del diámetro recristalizado calculado con la relación de Derby fue de 35,8 $\mu \mathrm{m}$, semejante al tamaño de grano determinado experimentalmente de $33,7 \mu \mathrm{m}$.

\section{CONCLUSIONES}

- Para el rango de temperaturas y velocidades de deformación estudiadas, donde se obtuvo un comportamiento de recristalización de picos múltiples, el modelo cosenoidal amortiguado de Avrami arrojó un comportamiento de tensión en función de la deformación próximo al experimentado por el cobre ETP durante su compresión con temperatura descendente. Esto sugiere que la aplicación de este modelo puede ser extendida a procesos de recristalización dinámica de picos múltiples con temperatura variable.

- La ecuación Universal de Derby arrojó valores de diámetros de granos recristalizados próximos a los medidos experimentalmente, para el rango de temperaturas y velocidades de deformación estudiadas, con una diferencia máxima entre ellos de 5,9\%.

\section{AGRADECIMIENTOS}

Queremos agradecer al Decanato de Investigación y Desarrollo de la Universidad Simón Bolívar (USB) 
por su contribución financiera a este proyecto; a todo el personal del Laboratorio E de la USB por su apoyo, en particular al TSU Henry López, y a los profesores Héber D’Armas y Verónica Di Graci por su colaboración.

\section{REFERENCIAS}

[1] P. Follansbee y U. Kocks, Acta Metall. 36 (1988) 81-93.

[2] V. García, J.M. Cabrera, L. Riera y J.M. Prado, Proceeding of Euromat 2000, Vol. 2, 2000, pp. 1.357-1.362.

[3] V. García, M. Wahabi, J.M. Cabrera, L. Riera y J.M. Prado, Rev. Metal. Madrid 37 (2001) 177-183.

[4] M. Avrami, J. Chem. Phys. 7 (1939) 1.103-1.112.

[5] M. Luton y C. Sellar, Acta Metall. 17 (1969) 1.033-1.043.

[6] A. Kolmogorov, Izv Akad, Nauk SSSR, Ser. Matem. 3 (1937) 355-359.

[7] W. Johnson y R. Mehl, Trans. AIME 135 (1939) 416-442.

[8] M. Wahabi, Tesis Doctoral, Dpto. de Ciencia de Materiales e Ingeniería Metalúrgica, Universidad Politécnica de Cataluña, 2002.

[9] I. Rieiro, M. Larrea, V. Gutiérrez, V. Triviño, M. Carsí y O. Ruano, Rev. Metal. Madrid 46 № Extraord. (2010) 115-127.

[10] Y. Prasad y K. Rao, Mat. Sci. Eng. A 317 (2004) 335-341.

[11] L. Blaz, T. Sakai y J. Jonas, Metal. Science, 17 (1983) 609-616.

[12] A. Manonukul y F. Dunne, Acta Mater. 47 (1999) 4.339-4.354.

[13] U. Kocks, J. Eng. Mater-T (Trans. ASME series H) 98 (1976) 76-85.

[14] A. Tanner y D. Mcdowell, Int. J. Plasticity 15 (1999) 375-399.

[15] A. Jena, M. Chaturvedi, Phase Transformations in Materials, (Ed.) Prentice Hall, 1992, pp. 243-247.

[16] V. García, Tesis Doctoral, Dpto. de Ciencia de Materiales e Ingeniería Metalúrgica, Universidad Politécnica de Cataluña, 2004.
[17] V. García, J. M. Cabrera, J. M. Prado, Mater. Sci. Forum, 550 (2007) 565-570.

[18] J.M. Cabrera, A. Omar y J. Prack, Rev. Metal. Madrid, 33 (1997) 143-152.

[19] A. Omar, A. Chenaoui, R. Dkiouak y J.M. Prado, Rev. Metal. Madrid 42 (2006) 103-113.

[20] B. Derby, Acta Metall. 39(1991) 955-962.

[21] G. Dieter, Metalurgia Mecánica, (Ed.) McGrawHill, 1967, pp. 510-519.

[22] Z. Zeng, S. Jonsson, H. Jorgen y Y. Zhang, Mater. Design. 30 (2009) 1.939-1.943.

[23] A. Rollett, D. Srolovitz, R. Doherty y M. Anderson, Acta Metall. 31 (1989) 627-439.

[24] R. Cahn y P. Haasen, Physical Metallurgy (1996) 2.452-2.453.

[25] J.M. Cabrera, Tesis Doctoral, Dpto. de Ciencia de Materiales e Ingeniería Metalúrgica, Universidad Politécnica de Cataluña, 1995.

[26] ASTM Standard E-209-00, Standard Practice for Compression Tests of Metallic Materials at Elevated Temperatures with Conventional or Rapid Heating Rates and Strain Rates, Vol. 03.01, ASTM International, 2010.

[27] ASTM E 3-11, Standard Guide for Preparation of Metallographic Specimens, Vol. 03.01, ASTM International, 2010.

[28] ASTM E 9-98a, Standard Test Methods of Tension Testing of Metallic Materials at room temperature, Vol. 03-01 ASTM Standards, 2010.

[29] J. Ruan y B. Bhushan, J. Appl. Phys. 76 (1994) 8.117-8.120.

[30] J. Peterson y B. Marshal, Technical Note 3657. National Advisory Committee for Aeronautics NACA, 1956.

[31] ASTM E 112-88, Standard Test Method for Determining the Average Grain Size, Vol. 02.01 ASTM Standards, 1992.

[32] S. Sakui, S. Sakai y K. Takeishi, Trans. ISIJ. 17 (1977) 718-725.

[33] F. Montheillet y J. Jonas, Encyclopedia of Applied Physics, 16 (1996) 205-225. 